\title{
Intense remodeling of extracellular matrix within the varicose vein: the role of gelatinases and vascular endothelial growth factor
}

\author{
Anna Horecka ${ }^{1}$ (D) - Anna Hordyjewska ${ }^{1}$ - Jadwiga Biernacka ${ }^{2}$ - Wojciech Dąbrowski ${ }^{2}$. Tomasz Zubilewicz ${ }^{3}$. \\ Agnieszka Malec ${ }^{4} \cdot$ Irena Musik $^{1} \cdot$ Jacek Kurzepa ${ }^{1}$
}

Received: 29 April 2020 / Accepted: 19 June 2020 / Published online: 27 June 2020

(C) The Author(s) 2020

\begin{abstract}
Background Increased blood pressure in the varicose veins (VV) can contribute to the overexpression of matrix metalloproteinases (MMPs), affecting the endothelium, smooth muscle, and extracellular matrix of the vein wall. Gelatinases (MMP-2 and MMP-9), hypoxia, and inflammation occurring in the VV wall contribute to the increased expression of vascular endothelial growth factor (VEGF).

Aims Our objective was to analyze the concentration of gelatinases and VEGF in the great saphenous VV wall and plasma of patients.

Methods In total, 65 patients (2nd degree according to clinical state classification, etiology, anatomy, and pathophysiologyCEAP classification) aged 22 to 70 were enrolled. Control veins $(n=10)$ were collected from the patients who underwent coronary artery bypass graft surgery. Control plasma $(n=20)$ was obtained from healthy individuals. Gelatinases and VEGF levels were measured with the usage of ELISA method.

Results A significant increase in MMP-9 (11.2 vs. $9.98 \mathrm{ng} / \mathrm{mg}$ of protein) and VEGF (41.06 vs. $26 \mathrm{ng} / \mathrm{g}$ of protein) concentration in VV wall compared with control veins was observed. A positive correlation between VEGF versus MMP-2 $(p=0.03, r=0.27)$ was found in the VV wall. However, no correlation was found between the concentration of VEGF and MMP-9 $(p=0.4, r=$ 0.11 ) in the VV wall. In addition, no statistical differences between MMP-9, MMP-2, and VEGF levels in plasma of VV patients compared with controls were noticed.

Conclusions The results of the present study confirm that VV's patients have altered expression of MMPs and VEGF. Overexpression of MMP-9 and VEGF in the VV wall may contribute to the spreading of inflammatory process and suggests the intense remodeling of extracellular tissue within the VV wall.
\end{abstract}

Keywords Gelatinases · Varicose veins · Vascular endothelial growth factor

\section{Introduction}

Anna Horecka

anna.horecka@umlub.pl

1 Department of Medical Chemistry, Medical University of Lublin, Chodźki 4A,, 20-093 Lublin, Poland

2 I Clinic of Anaesthesiology and Intensive Therapy with Clinical Paediatric Department, Medical University of Lublin, Jaczewskiego 8, 20-090 Lublin, Poland

3 Department of Vascular Surgery and Angiology, Medical University of Lublin, Staszica 17, 20-081 Lublin, Poland

4 Department of Obstetrics and Pathology of Pregnancy, Staszica 16, 20-081 Lublin, Poland
Chronic venous insufficiency (CVI) is an important medical problem in developed countries. Increased blood pressure in the varicose veins (VV) can contribute to the overexpression of selected matrix metalloproteinases (MMPs), affecting the endothelium, smooth muscle, and extracellular matrix proteins of the vein wall $[1,2]$. Gelatinases, which include MMP-2 (gelatinase A) and MMP-9 (gelatinase B), are responsible for the degradation of extracellular matrix (ECM) within the vein wall under both physiological and pathological conditions [3]. The main function of gelatinases involves degradation of fibers of denatured collagen but also the basement membrane and other structural components of ECM allowing migration of cells including smooth muscle cells [3, 4]. MMP2 is constitutively secreted by smooth muscle cells and 
vascular endothelial cells [5]. MMP-9 is present in large quantities in the granules of neutrophils. It plays a major role in the influx of leukocytes to the site of infection or damaged tissue during inflammatory processes [2].

Vascular endothelial growth factor (VEGF) stimulates the synthesis of MMPs, especially MMP-9 [5, 6]. Hypoxia and inflammation occurring in the VV wall contribute to the increased expression of VEGF in the connective tissues [7]. VEGF plays an important role in maintaining the integrity of blood vessel walls and during the process of angiogenesis [7]. It interferes with the integrity of the vascular wall and cell homeostasis by increasing the endothelial permeability [7]. This results in swelling and the formation of "fibrin cuffs" characteristic of CVI. In addition, VEGF activates endothelial nitric oxide synthase (eNOS), which dilates venous vessels [7, 8]. Impaired synthesis of VEGF may be a predictor of vascular diseases.

As the mechanisms leading to the formation of the VV are still not fully understood, the objective of our study was to analyze the concentration of gelatinases and VEGF in the VV wall and in the plasma of patients with $\mathrm{VV}$ as the potential agents involved in $\mathrm{VV}$ pathogenesis.

\section{Methods}

\section{Characteristics of the study group}

Sixty-five patients ( $\left.949, \delta^{\top} 16\right)$ aged 22 to 70 were enrolled (Table 1). The sample included patients with lower limb venous disease assigned as the 2nd degree according to clinical state classification, etiology, anatomy, and pathophysiology (CEAP), with varices present in the great saphenous vein [9]. Patients underwent VV surgery at the Institute of Rural Health in Lublin. The presence of thrombophlebitis or deep vein thrombosis, limb ischemia, clotting disorders, inflammatory diseases or cancer, diabetes mellitus, collagen diseases, surgical interventions in the last 12 months, and use of steroids and intravenous drugs in last 12 months excluded the patient from the study.

\section{Study material}

The material of VV patients was collected from femoral segment of varicose great saphenous vein using Babcock method and patients' blood was collected from the antecubital vein during surgery. Control groups consist of two subgroups. The first one includes control group no. $1\left(n=10,98, \delta^{\top} 2\right.$, aged 50 to 75$)$ patients who underwent coronary artery bypass graft (CABG) surgery, with no symptoms of VV and no retrograde flow proven by duplex ultrasound (DU) whose great saphenous veins were removed. The second one is control group no. $2(n=20, q 11,0$ 9 , aged 29 to 62) - healthy individuals whose blood was collected from the antecubital vein.

\section{Sample preparation}

Blood sample (VV and control no. 2) was collected from the antecubital vein into tubes with lithium heparin as an anticoagulant. Then it was centrifuged at $3000 \mathrm{rpm}$ to obtain plasma, in which gelatinases and VEGF concentration was determined. Next $0.5 \mathrm{~g}$ of venous walls (VV and control no. 1) was homogenized in $5 \mathrm{ml}$ of buffer containing $0.1 \mathrm{M}$ Tris- $\mathrm{HCl} \mathrm{pH} 7.4$ and centrifuged for $15 \mathrm{~min}$ at $3000 \mathrm{rpm}$. The prepared material was stored at temperature $-70^{\circ} \mathrm{C}$. Gelatinases and VEGF levels were measured in plasma and vein walls of $\mathrm{VV}$ patients and both control individuals (control no. 1 and control no. 2).

\section{Gelatinases and VEGF measurements}

Commercially available diagnostic kits, Human MMP-2 Quantikine ELISA Kit and Human MMP-9 Quantikine Immunoassay (R\&D System, Abingdon, UK), were applied to gelatin concentration evaluation. The gelatinase levels were expressed in nanograms per milliliter in plasma and in nanograms per milligram of protein in vein wall homogenates. VEGF concentration was determined using diagnostic kit Human VEGF Quantikine Immunoassay (R\&D System, Abingdon, UK) and expressed in picograms per milliliter in plasma, and nanograms per gram and nanograms per milligram of protein in tissue. Protein level in tissue samples was estimated with the usage of commercially available Bradford reagent (BIORAD Protein Assay, Hercules, USA). The assays were performed with the usage of Thermoshaker DTS-4 (ELMI, Calabasas, North America) and Microplate Reader Model 680 (BIO-RAD, Hercules, USA) with software Microplate Manager version 5.2.1 (BIO-RAD, Hercules, USA). All measurements were performed according to manufacturers' manuals.
Table 1 Characteristics of the study group

\begin{tabular}{|c|c|c|c|c|}
\hline & VV wall & Control 1 & Control 2 & $t$ test \\
\hline$n(\operatorname{sex})$ & $65(\uparrow 49$, ठૈ16) & $10(98$, ठ2) & $20(\uparrow 11, \overbrace{}^{\top} 9)$ & NA \\
\hline Age (years) & 22 to 70 & 50 to 75 & 29 to 62 & $p>0.05^{*}$ \\
\hline $\begin{array}{l}\text { Mean age } \pm \text { SD (years) } \\
\text { BMI }\end{array}$ & $\begin{array}{l}60 \pm 11.002^{*} \\
22.4 \pm 3.1^{*}\end{array}$ & $\begin{array}{l}62 \pm 8.3 \\
23.1 \pm 2.3\end{array}$ & $\begin{array}{l}56 \pm 10.2 \\
22.9 \pm 2.9\end{array}$ & \\
\hline Hypertension (yes/no) & $12 / 53$ & $4 / 6$ & $7 / 13$ & \\
\hline
\end{tabular}

*Difference between either VV wall group vs. control 1 or control 2 


\section{Statistical analysis}

$T$ test was applied in case of parametric distribution of values whereas the difference between values with non-parametric distribution was checked with Mann-Whitney $U$ test. Verification of hypothesis was performed at the significance level $\alpha=5 \%$ $(p<0.05)$. Values are expressed as mean $\pm \mathrm{SD}$ (Gaussian distribution) or median and 1st-3rd quartiles (non-Gaussian distribution). Spearman correlation coefficient has been used to establish the relationship between MMP-9, MMP-2, and VEGF concentration in VV wall. Statistical analysis was done with GraphPad InStat v. 3.10 (San Diego, USA).

\section{Results}

A significant increase in MMP-9 (11.2 vs. $9.98 \mathrm{ng} / \mathrm{mg}$ of protein) and VEGF (41.06 vs. $26 \mathrm{ng} / \mathrm{g}$ of protein) concentration in VV wall compared with control no. 1 was observed (Fig. 1). No statistical differences between MMP-9, MMP-2, and VEGF levels in plasma of VV patients were noticed compared with controls. A positive but weak correlation between the concentration of VEGF versus MMP-2 $(p=0.03, r=0.27)$ was found in the VV wall (Fig. 2). However, no correlation was found between the concentration of VEGF and MMP-9 $(p=0.4, r=0.11)$ in the VV wall.
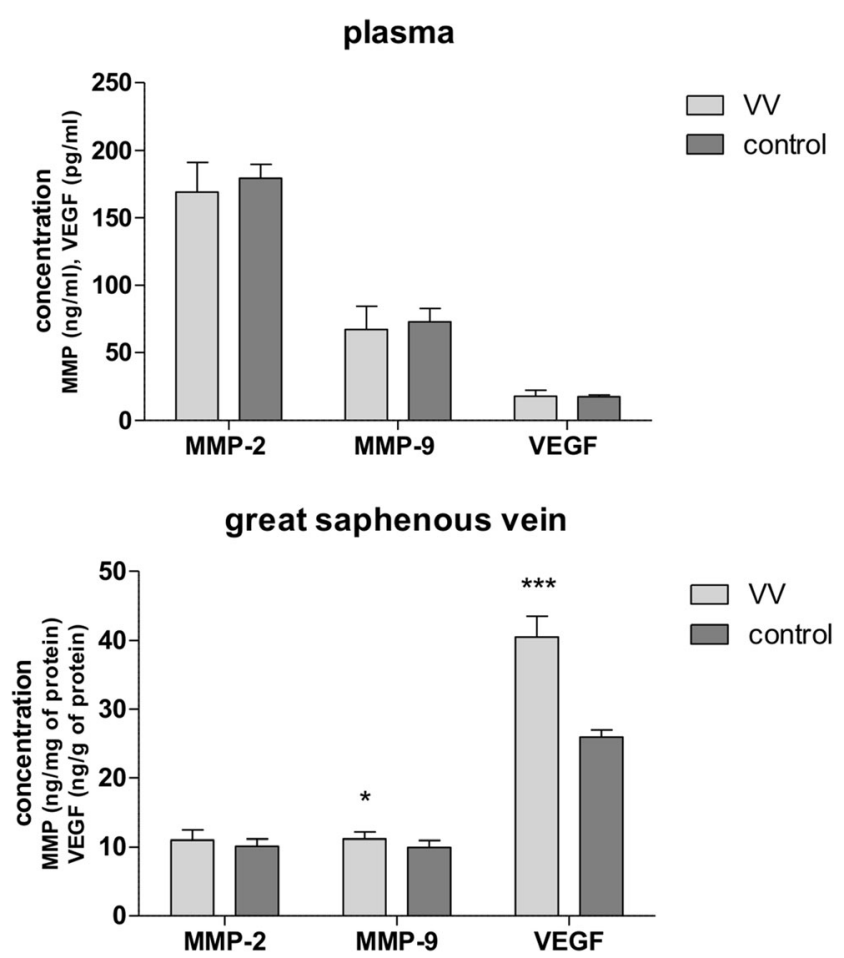

Fig. 1 Changes in concentration of gelatinases and VEGF in plasma and great saphenous veins of VV patients. Data are means (SD) or median (1st-3rd quartile); ${ }^{* *} p<0.001$, Mann-Whitney $U$ test; $* p<0.05, t$ test

\section{Discussion}

Changes in the activity of MMPs and VEGF were observed in many diseases of the circulatory system [10-13]. The VV development is associated with reduced wall thickness, changes in hemodynamics, the flow of inflammatory cytokines, changes in the ECM, and increased generation of reactive oxygen species (ROS) affecting the activity of MMPs [14-17]. Damaged epithelium causes the recruitment and adhesion of leukocytes, which play a significant role in the inflammatory process and lead to the damage of vessel walls. Prolonged oxidative stress in the VV wall is associated with the increased expression of gelatinases and hypoxia-inducible transcription factors (HIF): HIF- $1 \alpha$ responsible for the transcription of approximately 150 different genes including VEGF [5]. A positive MMP/TIMP ratio can cause excessive degradation of ECM leading to structural changes in the vein wall including valvular dysfunction that is one of the major factors affecting the development of $\mathrm{VV}[15,18]$. It was demonstrated that the patients with VV have the increased activity of several MMPs $(-1,-3,-13)$ in plasma and tissues $[19,20]$. The changes in the content of collagen in the VV wall, especially in the VV complicated by thrombophlebitis, can be caused by increased gelatinase activity $[21,22]$.

The previous results related to gelatinase activity in VV were ambiguous. The activity of MMP-2 was found to be decreased [23] or not changed [24-27] in VV wall in comparison with normal tissue. The increased activity of MMP-2 has been also found in the model of VV's cell line culture [28]. Our study revealed no statistically significant fluctuation of MMP-2 in the VV wall and plasma of patients. Its constitutive expression causes the MMP-2 level to be unsusceptible for pro-inflammatory cytokine fluctuation.

MMP-9 is important for maintaining the proper tension of blood vessel wall [2]. Serra et al. noticed the elevated level of MMP-9 and neutrophil gelatinase-associated lipocalin (NGAL) in the plasma of patients with venous ulcers [29]. Other reports demonstrated no changes $[26,30]$ in activity of MMP-9 or even decreased activity [31] in the VV. It was postulated that MMP-9 can be involved in the degradation of the vessel wall mainly of the media layer of vascular smooth muscle [29], whereas Huh et al. observed the increased expression of MMP-9 in endothelial cells of vascular smooth muscle [27]. In our study, a significant increase in MMP-9 concentration within VV wall compared with control no. 1 was observed. Higher MMP-9 level can be caused by elevated VEGF level, which is one of the most important inducers of MMP-9 expression. Overexpression of MMP-9 in the VV wall indicates the presence of the inflammatory process. This result supports the previous observations that MMP-9 can contribute to VV development [27]. The differences between MMP-9 level in study and control plasma were not statistically significant.

VEGF is considered to be the most potent stimulator of angiogenesis [32]. In addition, VEGF activates endothelial nitric oxide 
Fig. 2 A positive correlation between the concentration of VEGF vs. MMP-2 in the VV wall (Spearman correlation coefficient $p=0.03, r=0.27$ )

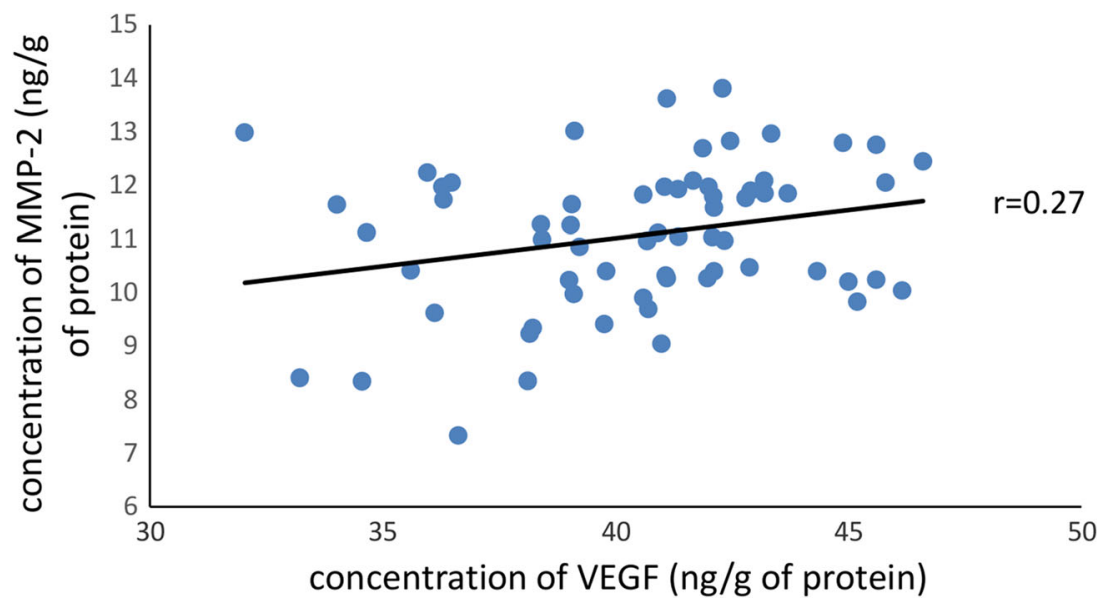

2 concentration in the VV wall. MMP-2 is a constitutive enzyme and VEGF directly affects its activation [19, 34, 37].

In conclusion, the results of the present study confirm that VV patients have altered expression of MMP-9 and VEGF. Overexpression of MMP-9 and VEGF in the VV wall may contribute to the spreading of inflammatory process and suggests the intense remodeling of extracellular tissue within the VV wall. The conducted study shows the coexistence of VV with elevated concentrations of VEGF and MMP-9 in the VV wall. The research does not determine whether it was the primary cause of VV or the increase in MMP-9 and VEGF.

Author contributors All authors contributed to the study conception and design. Material preparation, data collection, and analysis were performed by A.Horecka, A.Hordyjewska, J.B., W.D., T.Z., A.M., I.M., and J.K. The first draft of the manuscript was written by A.Horecka and all authors commented on previous versions of the manuscript. All authors read and approved the final manuscript.

Funding information The study was supported by Medical University of Lublin, Poland.

\section{Compliance with ethical standards}

Conflict of interest The authors declare that they have no competing interests.

Ethical approval The study protocol was approved by the Ethical Committee at the Medical University of Lublin (Poland), acceptance KE-0254/222/2009.

Informed consent All subjects enrolled into the study voluntarily agreed to participate in the study and signed an informed consent form before any study procedure in compliance with the Declaration of Helsinki.

Open Access This article is licensed under a Creative Commons Attribution 4.0 International License, which permits use, sharing, adaptation, distribution and reproduction in any medium or format, as long as you give appropriate credit to the original author(s) and the source, provide a link to the Creative Commons licence, and indicate if changes were made. The images or other third party material in this article are included 
in the article's Creative Commons licence, unless indicated otherwise in a credit line to the material. If material is not included in the article's Creative Commons licence and your intended use is not permitted by statutory regulation or exceeds the permitted use, you will need to obtain permission directly from the copyright holder. To view a copy of this licence, visit http://creativecommons.org/licenses/by/4.0/.

\section{References}

1. Barallobre-Barreiro J, Oklu R, Lynch M et al (2016) Extracellular matrix remodelling in response to venous hypertension: proteomics of human varicose veins. Cardiovasc Res 110:419-430

2. Xu J, Shi GP (2014) Vascular wall extracellular matrix proteins and vascular diseases. Biochim Biophys Acta 1842:2106-2119

3. Park H-J, Kim M-K, Kim Y et al (2017) Gastrin-releasing peptide promotes the migration of vascular smooth muscle cells through upregulation of matrix metalloproteinase- 2 and -9 . BMB Rep 50: 628-633

4. Lo Presti R, Hopps E, Caimi G (2017) Gelatinases and physical exercise: a systematic review of evidence from human studies. Medicine (Baltimore) 96:e8072

5. Raffetto JD, Khalil RA (2008) Mechanisms of varicose vein formation: valve dysfunction and wall dilation. Phlebology 23:85-98

6. Lim CS, Qiao X, Reslan O et al (2011) Prolonged mechanical stretch is associated with upregulation of hypoxia-inducible factors and reduced contraction in rat inferior vena cava. J Vasc Surg 53: 764-773

7. Koczy-Baron E, Kasperska-Zajac A (2014) The role of vascular endothelial growth factor in inflammatory processes. Postepy Hig Med Dosw 68:57-65

8. Kowalewski R, Małkowski A, Sobolewski K et al (2011) Vascular endothelial growth factor and its receptor in the varicose vein wall. Act Angiol 17:141-149

9. Eklöf B, Rutherford RB, Bergan JJ et al (2004) American Venous Forum International Ad Hoc Committee for Revision of the CEAP classification. Revision of the CEAP classification for chronic venous disorders: consensus statement. J Vasc Surg 40:1248-1252

10. Eelen G, de Zeeuw P, Simons M, Carmeliet P (2015) Endothelial cell metabolism in normal and diseased vasculature. Circ Res 116: 1231-1244

11. Humphrey JD, Milewicz DM (2017) Aging, smooth muscle vitality, and aortic integrity. Circ Res 120:1849-1851

12. Flórez A, De Haro J, Bleda S et al (2013) Analysis of vascular endothelial growth factor gene expression in the tissues of patients with chronic venous insufficiency. Phlebology 28:32-37

13. Benjamin MM, Khalil RA (2012) Matrix metalloproteinase inhibitors as investigative tools in the pathogenesis and management of vascular disease. EXS 103:209-279

14. Rafetto JD, Khalil RA (2008) Matrix metalloproteinases and their inhibitors in vascular remodeling and vascular disease. Biochem Pharmacol 75:346-359

15. Rafetto JD, Qiao X, Koledova VV et al (2008) Prolonged increases in vein wall tension increase matrix metalloproteinases and decrease constriction in rat vena cava: potential implications in varicose veins. J Vasc Surg 48:447-456

16. MacColl E, Khalil RA (2015) Matrix Metalloproteinases as regulators of vein structure and function: implications in chronic venous disease. J Pharmacol Exp Ther 355:410-428

17. Castro-Ferreira R, Cardoso R, Leite-Moreira A, Mansilha A (2018) The role of endothelial dysfunction and inflammation in chronic venous disease. Ann Vasc Surg 46:380-393
18. Kucukguven A, Khalil RA (2013) Matrix metalloproteinases as potential targets in the venous dilation associated with varicose veins. Curr Drug Targets 14:287-324

19. Fic P, Zakrocka I, Kurzepa J, Stepulak A (2011) Matrix metalloproteinases and atherosclerosis. Postepy Hig Med Dosw 65:16-27

20. Kurzawski M, Modrzejewski A, Pawlik A, Droździk M (2009) Polymorphism of matrix metalloproteinase genes (MMP1 and MMP3) in patients with varicose veins. Clin Exp Dermatol 34:613-617

21. Kowalewski R, Gacko M, Sobolewski K et al (2003) Gelatinases of varicose veins and varicose veins complicated by thrombophlebitis. Pol Prz Chir 75:947-955

22. Kowalewski R, Sobolewski K, Wolańska M et al (2004) Matrix metalloproteinases in the vein wall. Int Angiol 23:164-169

23. Badier-Commander C, Verneuren T, Lebard C et al (2000) Increased MMP/TIMP ratio in varicose veins: a possible explanation for extracellular matrix accumulation. J Pathol 192:105-112

24. Parra JR, Cambria RA, Hower CD et al (1998) Tissue inhibitor of metalloproteinase-1 is incerased in the saphenofemoral junction of patients with varices in the leg. J Vasc Surg 28:669-675

25. Sansilvestri-Morel P, Fioretti F, Rupin A et al (2007) Comparison of extracellular matrix in skin and saphenous veins from patients with varicose veins: does the skin reflect venous matrix changes? Clin Sci (Lond) 112:229-239

26. Irwin C, Synn A, Kraiss L, Zhang Q et al (2008) Metalloproteinase expression in venous aneurysms. J Vasc Surg 48:1278-1285

27. Huh S, Choi HH, Kim H-K et al (2010) The expression of matrix metalloproteinases and tissue inhibitors of metalloproteinases in the wall of great saphenous vein in patients with varicose veins. J Korean Surg Soc 79:16-25

28. Xiao Y, Huang Z, Yin $\mathrm{H}$ et al (2009) In vitro differences between smooth muscle cells derived from varicose veins and normal veins. J Vasc Surg 50:1149-1154

29. Serra R, Buffone G, Falcone D et al (2013) Chronic venous leg ulcers are associated with high levels of metalloproteinases- 9 and neutrophil gelatinase-associated lipocalin. Curr Drug Targets 14:287-324

30. Kosugi I, Urayama H, Kasashima F et al (2003) Matrix metalloproteinase-9 and urokinase-type plasminogen activator in varicose veins. Ann Vasc Surg 17:234-238

31. Woodside KJ, Hu M, Burke A et al (2003) Morphologic characteristics of varicose veins: possible role of metalloproteinases. J Vasc Surg 38:162-169

32. Johnson KE, Wilgus TA (2014) Vascular endothelial growth factor and angiogenesis in the regulation of cutaneous wound repair. Adv Wound Care (New Rochelle) 3:647-661

33. Hollingsworth SJ, Powell GL, Barker SGE (2005) Primary varicose veins: elevated plasma levels of the soluble isoform of the VEGF receptor flt-1 (s.flt-1). Phlebology 20:117-122

34. Wójcik E, Sas-Korczyńska B, Stasik Z et al (2010) MMP-9, TIMP-1 and VEGF in small cell lung cancer patients. Diag Lab 46:299-305

35. Alrehaili AA, Gharib AF, Karam RA et al (2020) Clinical significance of plasma MMP-2 and MMP-9 levels as biomarkers for tumor expression in breast cancer patients in Egypt. Mol Biol Rep 47: $1153-1160$

36. Abu El-Asrar AM, Mohammad G, Nawaz MI et al (2013) Relationship between vitreous levels of matrix metalloproteinases and vascular endothelial growth factor in proliferative diabetic retinopathy. PLoS One 8:e85857

37. Quintero-Fabián S, Arreola R, Becerril-Villanueva E et al (2019) Role of matrix metalloproteinases in angiogenesis and cancer. Front Oncol 9:1370

Publisher's note Springer Nature remains neutral with regard to jurisdictional claims in published maps and institutional affiliations. 